\title{
AVALIAÇÃO DO DESEMPENHO DE BICOS PARA APLICAÇÃO DE HERBICIDAS ${ }^{1}$
}

\author{
DILERMANDO PERECIN ${ }^{2}$, VALDEMIR A. PERESSIN ${ }^{3}$, TOMOMASSA MATUO $^{4}$, BENEDITO A. BRAZ ${ }^{5}$, LUIS C. PIO ${ }^{6}$
}

\section{RESUMO}

A avaliação do desempenho de barras de pulverização pode ser feita a partir do padrão de distribuição ou deposição do líquido de cada bico individual em mesa de prova. Neste trabalho comparam-se os padrões de deposição produzidos por diversos bicos em várias alturas. Os resultados mostraram melhores desempenho para os bicos TF e XR, seguidos pelos bicos TJ60-8010, TJ60-8006 e FL. Os bicos TJ60-11006 e os TQ mostraram problemas localizados e os bicos TK foram considerados os piores entre os testados. Relações entre o espaçamento entre bicos e altura do alvo para as quais os coeficientes de variação apresentam valores satisfatórios foram propostas.

Palavras chave: Tecnologia de aplicação, padrão de distribuição, barras de pulverização, mesa de prova.

\section{ABSTRACT}

\section{Nozzles and spray boom performance for herbicide application}

The performance of a spray boom can be evaluated from the individual nozzle distribution patterns determined in a patternator. The distribution patterns of several nozzle types at several heights were compared. Results showed better performances for TF and XR nozzles, followed by TJ60-8010, TJ60-8006 and FL nozzles. TJ60-11006 and TQ nozzles showed

\section{INTRODUÇÃO}

A uniformidade na distribuição da calda aplicada pela barra, em pulverizações de defensivos agrícolas, é dada pelas condições de montagem e de operação: espaçamento entre bicos, altura da barra, ângulo de abertura dos bicos e pressão de trabalho. O objetivo da pulverização é o de distribuir o herbicida selecionado de forma uniforme em toda área a ser tratada.

Os componentes fundamentais de qualquer sistema de pulverização são os bicos (Velini et al., 1995); todos os demais componentes some localized problems and TK nozzles presented the worst results.Combinations of the relation nozzle spacing and spray boom heights for satisfactory coefficient of variation were proposed.

Key words: Applying technology, distribution patterns, patternator.

${ }^{1}$ Recebido para publicação em 25/11/97 e na forma revisada em 07/08/98.

${ }^{2}$ Eng $^{\mathrm{o}}$. Agr ${ }^{\mathrm{o}}$., Prof. Titular, Dept ${ }^{\mathrm{o}}$ de Ciências Exatas, FCAVJ/UNESP. CEP 14870-000, Jaboticabal/SP.

${ }^{3} \mathrm{Eng}^{\circ}$. Agr ${ }^{\circ}$., Doutor, Pesquisador Div. Plantas Alimentícias Básicas/IAC/Campinas.

${ }^{4}$ Eng $^{\mathrm{o}}$. Agr ${ }^{\mathrm{o}}$., Prof. Titular, Dept ${ }^{\circ}$ de Defesa Fitossanitária. FCAVJ/UNESP. CEP 14870-000, Jaboticabal/SP.

${ }^{5} \mathrm{Eng}^{\mathrm{o}}$. Agr ${ }^{\circ}$., Doutor, Mestre em Produção Vegetal. FCAVJ/UNESP. CEP 14870-000, Jaboticabal/SP.

${ }^{6} \mathrm{Eng}^{\circ} \mathrm{Agr}^{\circ} /$ Herbicat Ltda./Catanduva. 
líquido na quantidade desejada por unidade de tempo e espalhar as gotas formadas dentro de uma área delimitada. Assim, a escolha incorreta dos bicos constitui-se numa das principais causas responsáveis por fracassos na aplicação de defensivos (Matuo, 1982).

$\mathrm{O}$ volume de calda aplicado em pulverização deve ser o mais uniforme possível, sob pena de exigir-se volumes adicionais para compensar os pontos ou faixas que recebem menores volumes. Entretanto, nas aplicações de herbicidas nem sempre isso pode ser feito, pois o excesso pode ser prejudicial.

Wolf \& Smith (1979), DeBoer \& Wiens (1983), Sartori (1985) sugeriram que um coeficiente de variação de 10 a $15 \%$ ou menos na distribuição da calda produz uma satisfatória uniformidade na aplicação . Roth et al. (1985) enfatizaram que a base para selecionar esses valores não foi discutida nos trabalhos publicados, devendo-se fundamentar em constatações práticoempíricas dos pesquisadores envolvidos. Provavelmente, há também uma certa dependência com o modo de ação do defensivo agrícola, bem como outros fatores relacionados à eficiência agrícola de cada produto. Assim, a uniformidade de aplicação está sujeita a algum grau de subjetividade.

Aspectos da tecnologia de aplicação, envolvendo o espaçamento dos bicos e altura de trabalho, podem ser encontrados na literatura, e foram discutidos em artigos anteriormente publicados por Matuo et al. (1994), Perecin et al. (1994), Peressin et al. (1996) e Perecin et al. (1998). Um ponto fundamental é a qualidade dos bicos e uma sistematização para essa avaliação a partir de resultados obtidos em mesa de prova pode ser encontrada em Perecin et al. (1994 e 1998).

Cada tipo de bico possui um padrão de distribuição característico, que determina a altura do bico em relação ao alvo, bem como o espaçamento dos mesmos numa barra de pulverização. $\mathrm{O}$ conhecimento da distribuição quantitativa da calda pulverizada ao longo da faixa de deposição, é, portanto, de grande importância nos estudos de pulverizações.

Esse trabalho tem como objetivo avaliar diferentes tipos de bicos através das vazões, do padrão de deposição em várias alturas do alvo e de propriedades que se obtém com a simulação da sobreposição dos bicos em barra de pulverização, em diferentes níveis de pressão de trabalho.

\section{MATERIAL E MÉTODOS}

\section{Levantamento dos dados com bicos isolados}

O trabalho foi desenvolvido em condições de laboratório da Faculdade de Ciências Agrárias e Veterinárias da Universidade Estadual Paulista Câmpus de Jaboticabal, incluindo a coleta de dados, produção dos padrões de deposição e simulação da pulverização em barra, com o auxílio de programas computacionais especialmente desenvolvidos para esse fim. São reunidos dados levantados em 55 situações, cada uma amostrando-se 10 exemplares de cada tipo de bico, entre os disponíveis no estoque de um distribuidor da Spraying Systems Co. (Herbicat Ltda) e englobando-se, além do tipo de bico, a pressão de trabalho e as alturas do alvo.

Coletaram-se os dados nas respectivas pressões por bico: TF-VS4 - $207 \mathrm{kPa}\left(30 \mathrm{lbf} / \mathrm{pol}^{2}\right)$; TJ60-11006 - $310 \mathrm{kPa}\left(45 \mathrm{lbf} / \mathrm{pol}^{2}\right)$; TJ60-8006 $310 \mathrm{kPa}$ (45 lbf/pol $\left.{ }^{2}\right)$; TJ60-8010 - $262 \mathrm{kPa}$ (38 lbf/pol'); TJ60-11010 - $276 \mathrm{kPa}\left(40 \mathrm{lbf} / \mathrm{pol}^{2}\right)$; TJ60-11006 - $241 \mathrm{kPa}\left(35 \mathrm{lbf} / \mathrm{pol}^{2}\right)$; TJ60-11010 $414 \mathrm{kPa}\left(60 \mathrm{lbf} / \mathrm{pol}^{2}\right)$; XR11006 - $327 \mathrm{kPa}$ (47,5lbf/pol $\left.{ }^{2}\right)$; FL-6,5 - $\left.290 \mathrm{kPa}(42 \mathrm{lbf} / \mathrm{pol})^{2}\right)$; FL-8 - $276 \mathrm{kPa}\left(40 \mathrm{lbf} / \mathrm{pol}^{2}\right) ; \mathrm{TK}-4 \quad-224 \mathrm{kPa}$ $\left(32,5 \mathrm{lbf} / \mathrm{pol}^{2}\right)$; TK-5 - $207 \mathrm{kPa}\left(30 \mathrm{lbf} / \mathrm{pol}^{2}\right)$; TK$\left.7,5-207 \mathrm{kPa}(30 \mathrm{lbf} / \mathrm{pol})^{2}\right)$ e TQ15006 - $327 \mathrm{kPa}$ $\left(47,5 \mathrm{lbf} / \mathrm{pol}^{2}\right)$. Para se avaliar a qualidade dos mesmos, determinou-se inicialmente a vazão, com os seus respectivos coeficientes de variação.

Em continuidade à avaliação da qualidade, determinou-se o padrão de distribuição da deposição em uma mesa de prova construída de acordo com as especificações da Organización Mundial de la Salud (1976) para testes de bicos. Essa mesa é constituída de uma chapa de metal corrugado formando canaletas distânciadas de $2,5 \mathrm{~cm}$ entre si, num total 
de 67 canaletas, tendo um tubo coletor graduado correspondendo a cada canaleta.

Cada bico a ser testado foi centrado na canaleta 33 e, avaliado no mínimo em três e no máximo em seis diferentes alturas, nas respectivas pressões, padronizado numa unidade de tempo (um minuto). Como foram amostrados 10 bicos de cada tipo, o levantamento foi repetido 10 vezes em cada altura (uma para cada bico), para cada uma das 55 situações estudadas.

Após a coleta dos dados em mesa de prova, sua transformação em dados percentuais por canaleta, elaborou-se o padrão de deposição de cada bico, em cada altura, através da construção de gráficos usando o SGPLUS. Os gráficos elaborados mostram a média percentual e respectivo intervalo de confiança por canaleta. Nove dos 55 gráficos construídos são mostrados na Figura 1, para ilustração.

Para resumir, de cada gráfico (média de 10 bicos amostrados) foram extraído o volume máximo porcentual depositado nas canaletas (VMAX), volume médio (VM) e desvio padrão (SM) dos porcentuais depositados nas canaletas passíveis de receber a calda, volume médio (VM23) e desvio padrão (SM23) dos porcentuais depositados nas 23 canaletas centrais. $\mathrm{O}$ efeito da altura sobre essas medidas, em 6 tipos de bicos, são mostrados na Figura 2.

\section{Simulação da barra de pulverização}

Utilizando a metodologia descrita em Perecin et al. (1998), as canaletas em cada situação e para cada tipo bico em teste foram combinadas com deslocamentos de $5 \mathrm{em} 5 \mathrm{~cm}$, simulando diferentes espaçamentos entre bicos na barra de pulverização. Os coeficientes de variação sistemáticos (CVS), determinados teoricamente, foram obtidos como os coeficientes de variação dos volumes compostos em cada nova posição ou canaleta. Como o processo foi repetido com 10 bicos, em cada teórica posição de $2,5 \mathrm{~cm}$ obtém-se um coeficiente de variação e a média desses coeficientes de variação foi tomada como coeficiente de variação aleatório (CVA). O coeficiente de variação global (CVG), teoricamente o esperado para a barra de pulverização, foi tomado usando a fórmula proposta por Perecin et al. (1998): $\mathrm{CVG}=\left[(\mathrm{CVS})^{2}+(\mathrm{CVA})^{2}\right]^{1 / 2}$.

$\mathrm{Na}$ Tabela 2, foram anotados os coeficientes de variação para os espaçamentos entre bicos, de 20,35, 50 e $65 \mathrm{~cm}$, que englobam as faixas normalmente usadas em barras de pulverização. Também foi constrúda a Tabela 3, selecionando-se as alturas $(\mathrm{H})$ e espaçamentos $(\mathrm{E})$ normalmente recomendados pelos fabricantes, mostando-se os CVG obtidos dentro dessas recomendações e qual seria o $\mathrm{E} / \mathrm{H}$ para obter o CVG próximo de $10 \%$, em cada altura $(\mathrm{H})$.

\section{RESULTADOS E DISCUSSÃO}

\section{Padrões de distribuição dos bicos}

A avaliação dos bicos hidráulicos foi realizada através da vazão e do padrão de distribuição dos mesmos, em diferentes alturas e em alguns casos com diferentes pressões.

Os padrões de distribuição ou de deposição nas 67 canaletas da mesa de prova, bem como os respectivos intervalos de confiança (segmento vertical), para cada uma das alturas, pressões e tipos de bicos, foram obtidos para cada tipo de bico, pressão de trabalho e altura do alvo, resultando 55 gráficos, dos quais 9 (nove) são apresentados na Figura 1, para ilustração. Gráficos em várias alturas, para o mesmo tipo e pressão de trabalho, foram mostrados em Perecin et al. (1998). Na Tabela 1 estão as estatísticas resumo das 55 situações e também as vazões proporcionadas pelos bicos num tempo de um minuto.

As vazões médias obtidas mostraram coeficientes de variação inferiores a 2,5 \% (Tabela 1), indicando que os bicos tem boa repetitividade de vazão. Matuo et al. (1994), Perecin et al. (1994), Peressin et al. (1996) e Perecin et al. (1998) também encontraram uma boa repetitividade no que se refere as vazões dos diferentes tipos de bicos avaliados, mostrando que a dificuldade da fabricação de bicos não está na confiabilidade de vazão mas na 

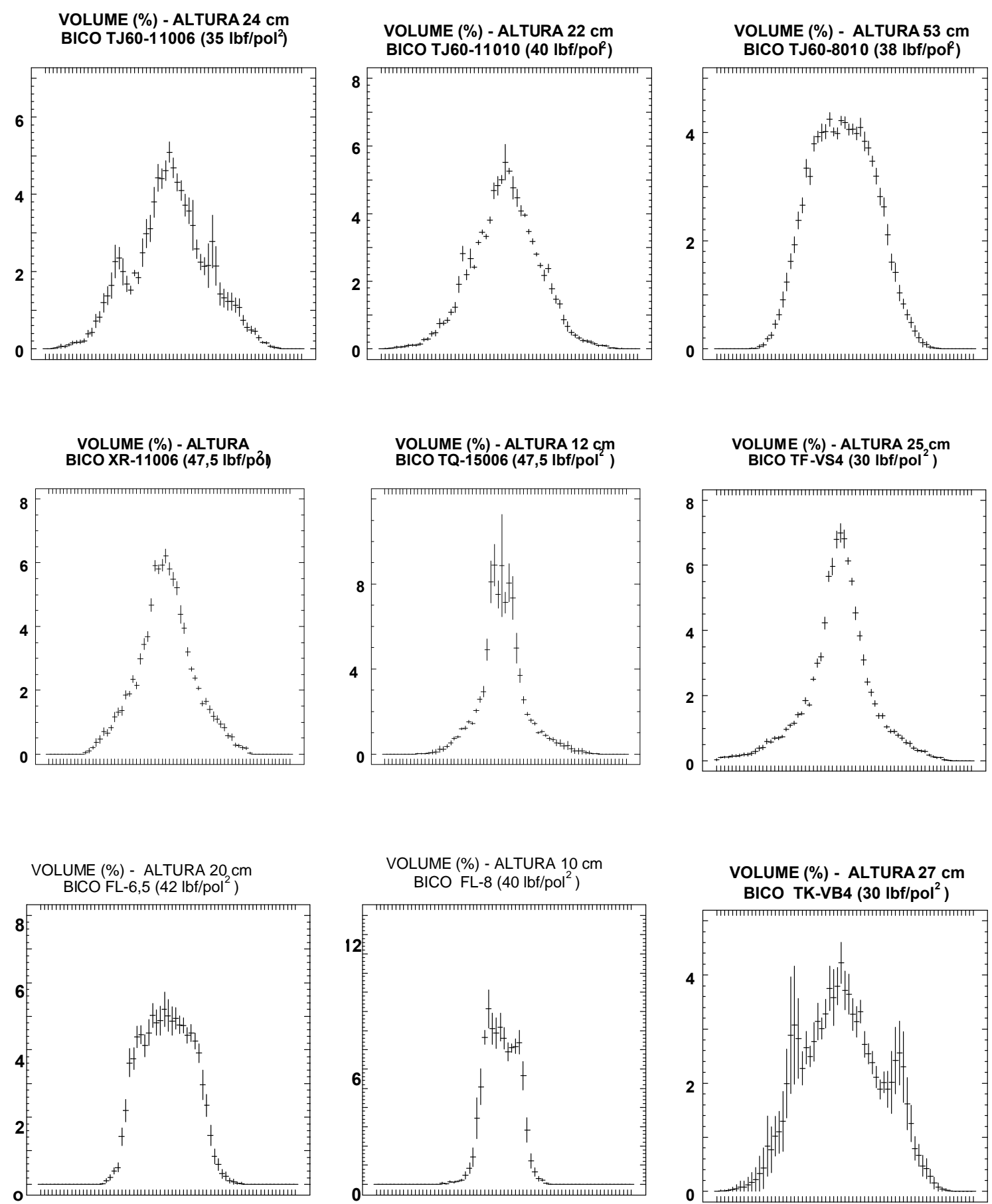

FIGURA 1. Padrões de distribuição de 9 tipos de bicos (um em cada gráfico), mostrando na ordenada o volume porcentual e respectivo intervalo de $95 \%$ de confiança (barra vertical), depositado em cada canaleta de $2,5 \mathrm{~cm}$ (indicada na abscissa). 

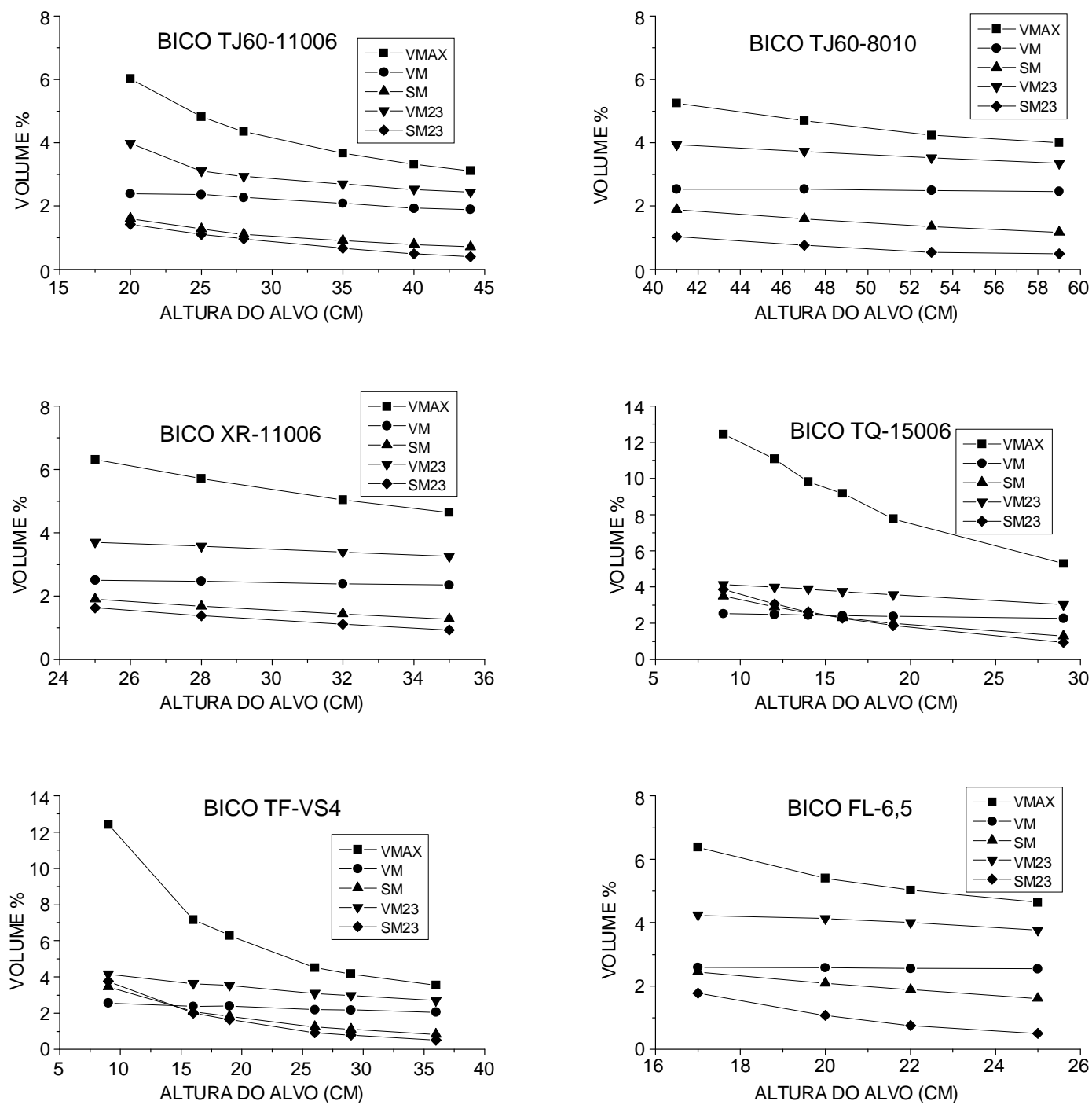

FIGURA 2. Efeito da altura do alvo em estatísticas resumo dos padrões de 6 tipos de bicos (média de 10 exemplares). $\mathrm{VMAX}=$ volumes máximos, $\mathrm{VM}=$ volumes médios, $\mathrm{SM}=$ desvios padrões, $\mathrm{VM} 23=$ volumes médios nas 23 canaletas centrais, SM23= desvios padrões nas 23 canaletas centrais. 
TABELA 1. Vazões dos bicos e estatísticas resumo dos padrões de distribuição da calda em diferentes alturas, para as 55 situações analisadas.

\begin{tabular}{|c|c|c|c|c|c|c|c|c|c|}
\hline \multirow[b]{2}{*}{ Bico } & \multirow{2}{*}{$\begin{array}{l}\text { Pressão } \\
\left(\mathrm{lbf} / \mathrm{pol}^{2}\right)\end{array}$} & \multicolumn{2}{|c|}{ Vazão (ml/min.) } & \multirow{2}{*}{$\begin{array}{l}\text { Altura } \\
(\mathrm{cm})\end{array}$} & \multicolumn{5}{|c|}{ Estatísticas resumo dos padrões $\left({ }^{1}\right)$} \\
\hline & & Média & C.V. (\%) & & VMAX & VM & SM & VM23 & SM23 \\
\hline \multirow[t]{3}{*}{ TJ60-11006 } & 35,0 & 1100,0 & 1,00 & 24 & 5,17 & 2,37 & 1,33 & 3,19 & 1,15 \\
\hline & & & & 27 & 4,67 & 2,31 & 1,12 & 3,00 & 0,99 \\
\hline & & & & 31 & 4,05 & 2,20 & 0,97 & 2,82 & 0,79 \\
\hline \multirow[t]{6}{*}{ TJ60-11006 } & 45,0 & 1167,8 & 0,86 & 20 & 6,02 & 2,38 & 1,60 & 3,98 & 1,42 \\
\hline & & & & 25 & 4,82 & 2,36 & 1,27 & 3,10 & 1,10 \\
\hline & & & & 28 & 4,35 & 2,27 & 1,10 & 2,93 & 0,96 \\
\hline & & & & 35 & 3,67 & 2,09 & 0,91 & 2,70 & 0,66 \\
\hline & & & & 40 & 3,32 & 1,93 & 0,78 & 2,52 & 0,49 \\
\hline & & & & 44 & 3,11 & 1,89 & 0,71 & 2,44 & 0,40 \\
\hline \multirow[t]{3}{*}{ TJ60-11010 } & 40,0 & 1670,0 & 1,13 & 22 & 5,53 & 2,41 & 1,62 & 2,41 & 1,08 \\
\hline & & & & 32 & 4,11 & 2,30 & 0,95 & 2,30 & 0,60 \\
\hline & & & & 42 & 3,35 & 2,09 & 0,66 & 2,09 & 0,37 \\
\hline \multirow[t]{3}{*}{ TJ60-11010 } & 60,0 & 2033,5 & 0,54 & 35 & 3,67 & 2,15 & 0,82 & 2,15 & 0,51 \\
\hline & & & & 45 & 3,05 & 2,00 & 0,61 & 2,00 & 0,35 \\
\hline & & & & 50 & 2,85 & 1,95 & 0,50 & 1,95 & 0,30 \\
\hline \multirow[t]{3}{*}{ TJ60-8010 } & 38,0 & 1648,5 & 0,90 & 53 & 4,37 & 2,50 & 1,48 & 3,64 & 0,60 \\
\hline & & & & 58 & 4,04 & 2,48 & 1,27 & 3,46 & 0,44 \\
\hline & & & & 63 & 3,72 & 2,46 & 1,09 & 3,30 & 0,34 \\
\hline \multirow[t]{4}{*}{ TJ60-8006 } & 45,0 & 1155,0 & 1,04 & 41 & 5,25 & 2,54 & 1,88 & 3,94 & 1,03 \\
\hline & & & & 47 & 4,70 & 2,53 & 1,59 & 3,72 & 0,76 \\
\hline & & & & 53 & 4,23 & 2,49 & 1,35 & 3,52 & 0,54 \\
\hline & & & & 59 & 4,00 & 2,46 & 1,17 & 3,35 & 0,49 \\
\hline \multirow[t]{4}{*}{ XR-11006 } & 47,5 & 1166,5 & 0,95 & 25 & 6,31 & 2,50 & 1,90 & 3,70 & 1,63 \\
\hline & & & & 28 & 5,71 & 2,47 & 1,68 & 3,57 & 1,38 \\
\hline & & & & 32 & 5,04 & 2,38 & 1,43 & 3,39 & 1,11 \\
\hline & & & & 35 & 4,64 & 2,35 & 1,27 & 3,25 & 0,93 \\
\hline
\end{tabular}


TABELA 1. Continuação.

\begin{tabular}{|c|c|c|c|c|c|c|c|c|c|}
\hline \multirow[b]{2}{*}{ Bico } & \multirow{2}{*}{$\begin{array}{l}\text { Pressão } \\
\left(\mathrm{lbf} / \mathrm{pol}^{2}\right)\end{array}$} & \multicolumn{2}{|c|}{ Vazão (ml/min.) } & \multirow{2}{*}{$\begin{array}{c}\text { Altura } \\
(\mathrm{cm})\end{array}$} & \multicolumn{5}{|c|}{ Estatísticas resumo dos padrões $\left({ }^{1}\right)$} \\
\hline & & Média & C.V. (\%) & & VMAX & VM & $\mathrm{SM}$ & VM23 & SM23 \\
\hline \multirow[t]{6}{*}{ TF-VS4 } & 30,0 & 1158,5 & 2,48 & 09 & 12,42 & 2,56 & 3,45 & 4,15 & 3,76 \\
\hline & & & & 16 & 7,16 & 2,37 & 2,09 & 3,64 & 2,01 \\
\hline & & & & 19 & 6,29 & 2,39 & 1,81 & 3,54 & 1,65 \\
\hline & & & & 26 & 4,51 & 2,19 & 1,24 & 3,09 & 0,91 \\
\hline & & & & 29 & 4,17 & 2,17 & 1,10 & 2,98 & 0,78 \\
\hline & & & & 36 & 3,53 & 2,06 & 0,82 & 2,69 & 0,50 \\
\hline \multirow[t]{6}{*}{ TQ-15006 } & 47,5 & 1168,7 & 1,07 & 09 & 12,44 & 2,53 & 3,50 & 4,14 & 3,87 \\
\hline & & & & 12 & 11,08 & 2,48 & 2,91 & 3,99 & 3,07 \\
\hline & & & & 14 & 9,80 & 2,46 & 2.57 & 3,89 & 2,62 \\
\hline & & & & 16 & 9,18 & 2,42 & 2,30 & 3,75 & 2,28 \\
\hline & & & & 19 & 7,77 & 2,37 & 1,98 & 3,58 & 1,87 \\
\hline & & & & 29 & 5,30 & 2,26 & 1,29 & 3,03 & 0,94 \\
\hline \multirow[t]{4}{*}{ FL-6,5 } & 42,0 & 1173,3 & 2,17 & 17 & 6,38 & 2,59 & 2,45 & 4,24 & 1,78 \\
\hline & & & & 20 & 5,40 & 2,58 & 2,09 & 4,13 & 1,07 \\
\hline & & & & 22 & 5,03 & 2,56 & 1,88 & 4,00 & 0,75 \\
\hline & & & & 25 & 4,64 & 2,55 & 1,60 & 3,76 & 0,50 \\
\hline \multirow[t]{4}{*}{ FL-8 } & 40,0 & 1159,5 & 2,17 & 09 & 10,27 & 10,27 & 3,62 & 4,32 & 3,80 \\
\hline & & & & 10 & 9,38 & 9,38 & 3,40 & 4,33 & 3,44 \\
\hline & & & & 12 & 7,90 & 7,90 & 3,01 & 4,32 & 2,78 \\
\hline & & & & 13 & 7,39 & 7,39 & 2,84 & 4,31 & 2,47 \\
\hline \multirow[t]{3}{*}{ TK-BV4 } & 32,5 & 1174,5 & 1,49 & 27 & 4,74 & 2,38 & 1,13 & 2,98 & 0,75 \\
\hline & & & & 37 & 3,58 & 2,13 & 0,66 & 2,43 & 0,48 \\
\hline & & & & 47 & 3,25 & 1,83 & 0,45 & 2,11 & 0,34 \\
\hline \multirow[t]{3}{*}{ TK-VB5 } & 30,0 & 1175,6 & 0,98 & 25 & 4,23 & 2,40 & 1,09 & 3,05 & 0,55 \\
\hline & & & & 35 & 3,22 & 2,13 & 0,57 & 2,38 & 0,34 \\
\hline & & & & 45 & 2,61 & 1,84 & 0,31 & 1,99 & 0,22 \\
\hline \multirow[t]{3}{*}{ TK-VB7,5 } & 30,0 & 1833,5 & 1,36 & 26 & 3,94 & 2,17 & 0,86 & 2,65 & 0,74 \\
\hline & & & & 36 & 3,24 & 1,84 & 0,66 & 2,25 & 0,50 \\
\hline & & & & 46 & 2,64 & 1,67 & 0,43 & 1,93 & 0,29 \\
\hline
\end{tabular}

( $\left.{ }^{1}\right)$ VMAX = volumes máximos; VM = volumes médios; $\mathrm{SM}=$ desvios padrões; VM23 = volumes médios nas 23 canaletas centrais; SM23 = desvios padrões nas 23 canaletas centrais. 
confiabilidade do padrão de distribuição.

Quanto as estatísticas resumo de cada gráfico (Tabela 1), nota-se que quase todas tendem a diminuir com a altura, o que é consequência do espalhamento. Como são médias de 10 exemplares em cada situação, há uma certa suavização, mostrando apenas maiores desvios padrões para os piores bicos ou situação inadequada de altura do alvo para o tipo de bico em questão. Conforme se pode observar na Figura 2, para seis dos 14 tipos de bicos, o efeito da altura é linear e praticamente paralelo para os volumes médios VM e VM23 e há uma pequena curvatura para os volumes máximos VMAX, como também para os desvios padrões SM e SM23, estes quase coincidentes, mostrando que há apenas maior espalhamento a medida que aumenta a altura do alvo, sem alterar muito o padrão (exceto pela pequena curvatura dos desvios padrões).

Os gráficos permitem análises mais detalhadas, mostrando as barras verticais que expressam a amplitude do intervalo de $95 \%$ de confiança, que indica a precisão dos bicos em cada canaleta ou posição.

Observa-se que os bicos TJ apresentaram picos nas laterais intermediárias do padrão, os quais foram mais evidentes no TJ6011006 , bem menos evidente no TJ60-11010 e inexistentes nos TJ60-8006 e TJ60-8010. Perecin et al. (1998) já tinha relatado este problema e sugeriu que o tipo de jato (jato plano duplo comum) pode ser o responsável por estes picos, não descartando uma possível contribuição devido a inclinação da mesa de prova ou mesmo problemas na fabricação dos bicos, principalmente nos TJ60-11006.

Os bicos XR, TF e FL foram os que apresentaram os melhores padrões, sem problemas localizados como os TJ e os TQ. Os TQ mostraram problemas nas canaletas centrais e uniformidade similar aos TF e XR em outras posições.

Finalmente, observa-se que os bicos TK foram os piores, além de apresentar de forma quase que generalizada uma maior variabilidade, mostraram variações de padrão quando se comparam os bicos TK-VB4, TK-VB5 e TKVB7,5.

Desta forma pode-se dizer que os melhores bicos foram os TF e os XR, seguidos pelos TJ60-8010, TJ60-8006, TJ60-11010 e pelos FL. No que se refere aos TJ60-11006 e aos TQ ambos apresentaram problemas localizados e os TK foram considerados os piores, entre os bicos avaliados.

Galli et al. (1983) constataram diferenças apreciáveis nos padrões de deposição entre alguns tipos de bicos e, recomendaram a realização de cuidadosos testes antes do seu emprego. Os resultados encontrados, no tocante aos bicos TK, TQ e TJ são preocupantes e evidênciam a necessidade da elaboração destes testes.

\section{Simulação dos bicos em barra de pulverização}

Com um programa construído para esse fim (Perecin et al., 1994), foram obtidos os CV para cada "teórica" posição da barra. Os espaçamentos entre bicos variaram de 2,5 a $125 \mathrm{~cm}$ em intervalos de $2,5 \mathrm{~cm}$, resultando um volume de dados impossível de ser apresentado. Na Tabela 2 estão os coeficientes de variação de barras de pulverização teoricamente simulados com espaçamentos de $20,35,50$ e $65 \mathrm{~cm}$ entre bicos, permitindo comparações e avaliações.

Além disso, em função das recomendações da Spraying Systems (1994) e das combinações de $\mathrm{E} / \mathrm{H}$ para atingir CVG similar a $10 \%$, elaborou-se a Tabela 3.

As barras de pulverização irão refletir as qualidades e defeitos do padrão do bico em cada situação. Embora Wolf \& Smith (1979), DeBoer \& Wiens (1983), Sartori (1985) tenham sugerido que um coeficiente de variação de 10 a $15 \%$ ou menos produza uma satisfatória uniformidade de distribuição do líquido pulverizado, Perecin et al. (1998) sugeriram para experimentos conduzidos em condições de laboratório que os CVG aceitáveis na prática devem ser até inferiores a $10 \%$, uma vez que, em condições de campo, estes valores tendem a aumentar, quer seja pelas 
condições climáticas ocorrentes por ocasião da aplicação ou pelos movimentos desordenados da barra de pulverização, conforme enfatizado por Nation (1982).

Notam-se que os CVA variam menos que os CVS, quando se alteram os espaçamentos entre bicos; pois eles dependem fundamentalmente da variação entre bicos, embora também sejam influenciados pela alteração no número de jatos que se superpõem ao se alterar o espaçamento entre bicos. Assim sendo, os CVA podem ser tomados como um padrão de qualidade dos bicos produzidos, notando-se então que entre os melhores bicos nesse aspecto estariam, por exemplo, os TF, XR, FL e alguns TJ e entre os piores estariam os TK e TQ, o que pode ser visto tomando-se, por exemplo, o espaçamento de 50 $\mathrm{cm}$ entre bicos na Tabela 2.

TABELA 2. Coeficientes de variação, sistemático (CVS), aleatório (CVA) e global (CVG) da barra de pulverização de alguns bicos, em função de espaçamentos (E) entre bicos, pressão de trabalho e altura do alvo.

\begin{tabular}{|c|c|c|c|c|c|c|c|c|c|c|c|c|c|c|}
\hline \multirow[b]{2}{*}{ Bico } & \multirow{2}{*}{$\begin{array}{l}\text { Pressão } \\
\text { lbf/pol }\end{array}$} & \multirow{2}{*}{$\begin{array}{l}\text { Altura } \\
\text { (cm) }\end{array}$} & \multicolumn{3}{|c|}{$E=20 \mathrm{~cm}$} & \multicolumn{3}{|c|}{$\mathrm{E}=35 \mathrm{~cm}$} & \multicolumn{3}{|c|}{$E=50 \mathrm{~cm}$} & \multicolumn{3}{|c|}{$E=65 \mathrm{~cm}$} \\
\hline & & & CVS & CVA & CVG & CVS & CVA & CVG & CVS & CVA & CVG & CVS & CVA & CVG \\
\hline \multirow[t]{3}{*}{ TJ60-11006 } & 35,0 & 24 & 4,6 & 5,8 & 7,4 & 14,1 & 9,4 & 16,9 & 15,2 & 9,1 & 17,8 & 18,4 & 13,8 & 23,0 \\
\hline & & 27 & 2,6 & 5,7 & 6,3 & 11,6 & 9,6 & 15,0 & 17,3 & 8,8 & 19,4 & 14,0 & 12,0 & 18,4 \\
\hline & & 31 & 7,5 & 6,2 & 9,8 & 7,3 & 8,8 & 11,4 & 20,3 & 9,9 & 22,6 & 13,0 & 10,2 & 16,5 \\
\hline \multirow[t]{6}{*}{ TJ60-11006 } & 45,0 & 20 & 3,6 & 4,2 & 5,5 & 18,0 & 7,1 & 19,3 & 18,6 & 10,1 & 21,2 & 27,9 & 13,7 & 31,2 \\
\hline & & 25 & 1,9 & 5,1 & 5,5 & 11,1 & 9,0 & 14,3 & 18,4 & 9,4 & 20,7 & 15,1 & 13,1 & 20,0 \\
\hline & & 28 & 4,0 & 4,7 & 6,1 & 4,9 & 8,7 & 10,0 & 20,7 & 10,0 & 23,0 & 13,8 & 11,6 & 18,0 \\
\hline & & 35 & 3,1 & 5,3 & 6,1 & 3,8 & 10,6 & 11,3 & 17,5 & 11,2 & 20,8 & 17,5 & 11,3 & 20,9 \\
\hline & & 40 & 1,5 & 4,5 & 4,7 & 8,0 & 6,4 & 10,2 & 10,6 & 9,9 & 14,5 & 22,8 & 9,0 & 24,5 \\
\hline & & 44 & 3,1 & 5,0 & 5,9 & 6,2 & 7,5 & 9,7 & 6,4 & 9,8 & 11,7 & 22,5 & 10,1 & 24,7 \\
\hline \multirow[t]{3}{*}{ TJ60-11010 } & 40,0 & 22 & 4,0 & 4,0 & 5,7 & 8,0 & 4,3 & 9,1 & 10,5 & 5,9 & 12,1 & 22,8 & 7,0 & 23,8 \\
\hline & & 32 & 3,5 & 5,0 & 6,1 & 3,7 & 5,4 & 6,5 & 7,6 & 5,5 & 9,4 & 6,7 & 6,7 & 9,5 \\
\hline & & 42 & 2,6 & 4,2 & 5,0 & 3,1 & 5,1 & 6,0 & 7,6 & 6,7 & 10,1 & 6,7 & 5,9 & 8,9 \\
\hline \multirow[t]{3}{*}{ TJ60-11010 } & 60,0 & 35 & 2,3 & 3,8 & 4,4 & 3,2 & 5,6 & 6,4 & 9,1 & 6,1 & 10,9 & 9,7 & 6,6 & 11,7 \\
\hline & & 45 & 1,4 & 3,0 & 3,3 & 5,1 & 5,2 & 7,3 & 3,9 & 4,5 & 5,9 & 11,4 & 5,5 & 12,7 \\
\hline & & 50 & 1,8 & 3,8 & 4,2 & 6,9 & 5,8 & 9,0 & 3,8 & 5,9 & 7,0 & 10,0 & 6,1 & 11,7 \\
\hline \multirow[t]{3}{*}{ TJ60-8010 } & 38,0 & 53 & 1,4 & 2,6 & 3,0 & 5,2 & 4,2 & 6,7 & 9,2 & 4,9 & 10,5 & 8,3 & 4,9 & 9,7 \\
\hline & & 58 & 1,2 & 2,1 & 2,4 & 3,8 & 2,8 & 4,7 & 10,0 & 5,4 & 11,4 & 3,5 & 4,8 & 5,9 \\
\hline & & 63 & 1,2 & 2,3 & 2,6 & 2,9 & 3,5 & 4,5 & 10,0 & 4,6 & 11,0 & 5,9 & 4,5 & 7,4 \\
\hline \multirow[t]{4}{*}{ TJ60-8006 } & 45,0 & 41 & 1,6 & 3,2 & 3,6 & 6,7 & 6,9 & 9,6 & 2,8 & 6,3 & 6,9 & 25,7 & 8,7 & 27,2 \\
\hline & & 47 & 1,8 & 3,1 & 3,6 & 4,5 & 5,8 & 7,4 & 5,9 & 6,8 & 9,0 & 13,4 & 7,0 & 15,1 \\
\hline & & 53 & 1,9 & 2,7 & 3,3 & 4,0 & 5,4 & 6,7 & 8,4 & 7,8 & 11,5 & 5,4 & 7,1 & 8,9 \\
\hline & & 59 & 2,3 & 3,8 & 4,4 & 3,9 & 5,4 & 6,7 & 7,5 & 8,9 & 11,6 & 4,4 & 8,0 & 9,2 \\
\hline \multirow[t]{4}{*}{ XR-11006 } & 47,5 & 25 & 3,3 & 2,9 & 4,4 & 13,2 & 4,9 & 14,0 & 22,3 & 5,8 & 23,0 & 35,1 & 7,2 & 35,8 \\
\hline & & 28 & 3,2 & 3,2 & 4,6 & 9,2 & 5,2 & 10,6 & 18,9 & 5,1 & 19,5 & 27,1 & 7,4 & 28,1 \\
\hline & & 32 & 2,8 & 3,1 & 4,2 & 4,4 & 5,2 & 8,9 & 15,6 & 5,2 & 16,5 & 19,7 & 7,2 & 21,0 \\
\hline & & 35 & 2,3 & 3,8 & 4,4 & 3,2 & 5,4 & 6,3 & 13,9 & 6,0 & 15,2 & 16,3 & 6,6 & 17,6 \\
\hline \multirow[t]{6}{*}{ TF- VS4 } & 30,0 & 09 & 20,6 & 4,3 & 21,0 & 48,4 & 5,7 & 48,7 & 71,7 & 7,1 & 72,1 & 94,2 & 9,6 & 94,7 \\
\hline & & 16 & 3,0 & 4,0 & 5,0 & 18,1 & 4,7 & 18,7 & 34,4 & 5,7 & 34,9 & 47,9 & 6,5 & 48,4 \\
\hline & & 19 & 2,9 & 4,0 & 5,0 & 11,4 & 4,8 & 12,4 & 27,6 & 5,4 & 28,2 & 35,7 & 5,9 & 36,2 \\
\hline & & 26 & 1,7 & 4,3 & 4,6 & 3,5 & 4,9 & 6,0 & 13,1 & 5,7 & 14,2 & 23,1 & 6,3 & 23,9 \\
\hline & & 29 & 1,9 & 4,5 & 4,9 & 3,1 & 5,0 & 5,9 & 10,8 & 5,1 & 12,0 & 19,8 & 5,6 & 20,6 \\
\hline & & 36 & 2,2 & 4,1 & 4,7 & 3,8 & 4,5 & 5,9 & 4,2 & 4,9 & 6,5 & 14,3 & 5,2 & 15,2 \\
\hline
\end{tabular}


TABELA 2. Continuação.

\begin{tabular}{|c|c|c|c|c|c|c|c|c|c|c|c|c|c|c|}
\hline \multirow[b]{2}{*}{ Bico } & \multirow{2}{*}{$\begin{array}{l}\text { Pressão } \\
{\text { lbf } / \text { pol }^{2}}^{2}\end{array}$} & \multirow{2}{*}{$\begin{array}{l}\text { Altura } \\
\text { (cm) }\end{array}$} & \multicolumn{3}{|c|}{$E=20 \mathrm{~cm}$} & \multicolumn{3}{|c|}{$\mathrm{E}=35 \mathrm{~cm}$} & \multicolumn{3}{|c|}{$E=50 \mathrm{~cm}$} & \multicolumn{3}{|c|}{$E=65 \mathrm{~cm}$} \\
\hline & & & CVS & CVA & CVG & CVS & CVA & CVG & CVS & CVA & CVG & CVS & CVA & CVG \\
\hline \multirow[t]{6}{*}{ TQ-15006 } & 47,5 & 09 & 12,2 & 12,6 & 17,5 & 47,9 & 13,7 & 49,8 & 71,2 & 15,6 & 72,8 & 93,7 & 19,2 & 95,6 \\
\hline & & 12 & 7,3 & 12,2 & 14,2 & 32,7 & 11,8 & 34,7 & 52,7 & 11,3 & 53,9 & 72,2 & 14,3 & 73,6 \\
\hline & & 14 & 7,7 & 12,2 & 14,4 & 23,4 & 12,5 & 26,5 & 42,9 & 12,4 & 44,7 & 59,7 & 15,0 & 61,6 \\
\hline & & 16 & 8,2 & 11,3 & 14,0 & 17,6 & 10,2 & 20,3 & 37,2 & 10,1 & 38,5 & 50,3 & 10,7 & 51,4 \\
\hline & & 19 & 8,3 & 11,3 & 14,0 & 10,5 & 12,8 & 16,5 & 28,0 & 13,3 & 31,0 & 39,4 & 12,9 & 41,4 \\
\hline & & 29 & 3,4 & 6,9 & 7,7 & 7,8 & 13,3 & 15,4 & 9,6 & 14,6 & 17,5 & 19,4 & 15,9 & 25,1 \\
\hline \multirow[t]{4}{*}{ FL- 6,5} & 42,0 & 17 & 6,4 & 5,5 & 8,4 & 15,1 & 10,1 & 18,1 & 16,9 & 13,6 & 21,7 & 50,9 & 21,0 & 55,0 \\
\hline & & 20 & 6,9 & 3,8 & 7,8 & 16,3 & 6,9 & 17,7 & 6,4 & 10,8 & 12,6 & 33,1 & 14,9 & 36,3 \\
\hline & & 22 & 4,0 & 3,8 & 5,5 & 12,8 & 7,4 & 14,6 & 12,9 & 9,8 & 16,2 & 21,9 & 12,0 & 24,9 \\
\hline & & 25 & 2,8 & 3,5 & 4,5 & 6,8 & 6,1 & 9,1 & 18,9 & 11,0 & 21,8 & 7,7 & 11,8 & 14,1 \\
\hline \multirow[t]{4}{*}{ FL- 8} & 40,0 & 9 & 21,7 & 12,8 & 25,2 & 30,9 & 19,3 & 36,5 & 70,7 & 27,9 & 76,0 & 97,3 & 35,9 & 103,7 \\
\hline & & 10 & 19,8 & 11,4 & 22,9 & 19,7 & 16,4 & 25,6 & 62,0 & 25,6 & 67,1 & 89,4 & 34,6 & 95,9 \\
\hline & & 12 & 9,8 & 11,0 & 14,7 & 9,6 & 16,0 & 18,6 & 44,6 & 22,0 & 49,7 & 74,2 & 29,4 & 79,9 \\
\hline & & 13 & 2,6 & 8,8 & 9,1 & 18,4 & 13,4 & 22,7 & 36,3 & 18,7 & 40,8 & 67,5 & 25,2 & 72,1 \\
\hline \multirow[t]{3}{*}{ TK-VB4 } & 32,5 & 27 & 3,8 & 8,2 & 9,1 & 15,5 & 13,4 & 20,4 & 9,3 & 18,3 & 20,5 & 16,9 & 17,8 & 24,5 \\
\hline & & 37 & 3,2 & 8,8 & 9,4 & 5,5 & 13,0 & 14,1 & 17,4 & 14,3 & 22,6 & 11,4 & 19,2 & 22,4 \\
\hline & & 47 & 47 & 7,0 & 8,0 & 6,9 & 10,3 & 12,4 & 10,5 & 16,3 & 19,4 & 14,2 & 14,4 & 20,3 \\
\hline \multirow[t]{3}{*}{ TK-VB5 } & 30,0 & 25 & 3,7 & 9,1 & 9,8 & 10,8 & 15,2 & 18,7 & 9,0 & 17,0 & 19,2 & 13,9 & 19,7 & 24,1 \\
\hline & & 35 & 1,1 & 7,5 & 7,6 & 5,7 & 11,3 & 12,6 & 10,8 & 15,4 & 18,9 & 9,2 & 17,2 & 19,5 \\
\hline & & 45 & 1,2 & 6,4 & 6,5 & 3,3 & 9,2 & 9,8 & 7,1 & 13,5 & 15,2 & 11,2 & 12,1 & 16,5 \\
\hline TK- & 30,0 & 26 & 4,0 & 38,5 & 38,7 & 5,4 & 38,5 & 38,9 & 13,3 & 42,3 & 44,3 & 11,6 & 40,6 & 42,3 \\
\hline \multirow[t]{2}{*}{ VB7,5 } & & 36 & 2,9 & 37,7 & 37,8 & 4,7 & 38,5 & 38,8 & 6,8 & 39,3 & 39,9 & 20,6 & 38,9 & 44,1 \\
\hline & & 46 & 2,4 & 6,9 & 7,3 & 9,9 & 8,6 & 13,1 & 12,7 & 9,0 & 15,6 & 19,7 & 12,6 & 23,4 \\
\hline
\end{tabular}

Por sua vez, os CVS dependem fundamentalmente do espaçamento entre bicos e os CVG, consequência de ambos. A Tabela 2 permite, pelo menos de forma aproximada, escolher as condições desejadas de espaçamento entre bicos e altura do alvo para não superar um determinado CVG.

Por outro lado, na Tabela 3 observa-se que a relação $\mathrm{E} / \mathrm{H}$ fornecida pelo o fabricante nem sempre é a mais recomendada. Existem vários exemplos:

-1) no caso do bico TJ60-11006 (E/H do fabricante $=1,25)$, na pressão de $35 \mathrm{lbf} / \mathrm{pol}^{2} \mathrm{com} \mathrm{E} / \mathrm{H}=1,13$ resulta $\mathrm{CVG}=11,4$, ou seja, o $\mathrm{E} / \mathrm{H}$ deveria ser menor que o recomendado; já para 45,0 lbf/ $\mathrm{pol}^{2}$ com $\mathrm{E} / \mathrm{H}=1,25$ resultam $\mathrm{CVG}$ variando de $10 \mathrm{a}$ 14,5 , sugerindo um provável efeito da pressão melhorando o padrão do bico; também cabe enfatizar que os outros TJ tiveram melhor comportamento;

- 2) os valores de $\mathrm{E} / \mathrm{H}$ do fabricante $=1,875$, para os bicos TF e FL devem ser reavaliados, uma vez que produzem sempre CVG superiores a $10 \%$;
- 3) para os bicos TK há necessidade de $\mathrm{E} / \mathrm{H}$ inferiores a 1 para se produzir CVG similares a $10 \%$, mostrando ser difícil atingir essa precisão com esses bicos, nas alturas usadas na prática.

Uma boa discussão sobre a importância da relação $\mathrm{E} / \mathrm{H}$, bem como uma visualização gráfica, pode ser encontrada em Matuo et al. (1994) e Peressin et al. (1996), cabendo enfatizar que trabalhos desta natureza são importantes para obtenção de melhorias na qualidade de aplicação. Os dados deste trabalho, por exemplo, evidenciam que a relação $\mathrm{E} / \mathrm{H}$, principalmente para os bicos TF e FL, devem ser reavaliadas pelos fabricantes.

\section{LITERATURA CITADA}

DeBOER, L.J.; WIENS, E.H. Test procedures for granular applicator performance testing. St. Joseph, MI: ASAE, 1983. (ASAE Paper $\mathrm{N}^{\circ}$ 83-1504). 
TABELA 3. Coeficientes de variação global $(\mathrm{CVG})$ da barra de pulverização de alguns bicos, em função de espaçamentos (E) entre bicos, pressão de trabalho e altura do alvo $(\mathrm{H})$, para algumas situações de maior interesse.

\begin{tabular}{|c|c|c|c|c|c|c|c|}
\hline \multirow[t]{2}{*}{ Bico } & \multirow{2}{*}{$\begin{array}{l}\text { Pressão } \\
\left(\mathrm{lbf} / \mathrm{pol}^{2}\right)\end{array}$} & \multirow{2}{*}{$\begin{array}{c}\text { Altura }(\mathrm{H}) \\
\mathrm{H}(\mathrm{cm})\end{array}$} & \multirow{2}{*}{$\begin{array}{c}\text { Espaçamento } \\
\text { E }(\mathrm{cm})\end{array}$} & \multicolumn{2}{|c|}{ OBTIDOS } & \multirow{2}{*}{$\begin{array}{c}\text { RECOMENDADO } \\
\text { E/H }\left(^{1}\right)\end{array}$} & \multirow{2}{*}{$\begin{array}{c}\text { PARA CVG } \cong 10 \% \\
\mathrm{E} / \mathrm{H}\end{array}$} \\
\hline & & & & $\mathrm{CVG}$ & $\mathrm{E} / \mathrm{H}$ & & \\
\hline \multirow[t]{2}{*}{ TJ60-11006 } & 35,0 & 27 & 35 & 15,0 & 1,30 & 1,25 & 1,11 \\
\hline & & 31 & 35 & 11,4 & 1,13 & & 1,00 \\
\hline \multirow{2}{*}{ TJ60-11006 } & 45,0 & 28 & 35 & 10,0 & 1,25 & 1,25 & 1,25 \\
\hline & & 40 & 50 & 14,5 & 1,25 & & 1,00 \\
\hline \multirow{2}{*}{ TJ60-11010 } & 40,0 & 32 & 50 & 9,4 & 1,56 & 1,25 & 1,25 \\
\hline & & 42 & 50 & 10,1 & 1,19 & & 1,20 \\
\hline \multirow[t]{2}{*}{ TJ60-11010 } & 60,0 & 35 & 50 & 10,9 & 1,43 & 1,25 & 1,37 \\
\hline & & 45 & 50 & 5,9 & 1,11 & & 1,20 \\
\hline \multirow[t]{2}{*}{ TJ60-8010 } & 38,0 & 53 & 50 & 10,5 & 0,94 & 1,00 & 1,23 \\
\hline & & 53 & 35 & 6,7 & 0,66 & & 1,21 \\
\hline \multirow[t]{2}{*}{ TJ60-8006 } & 45,0 & 53 & 50 & 11,5 & 0,94 & 1,00 & 1,23 \\
\hline & & 47 & 50 & 9,0 & 1,06 & & 1,28 \\
\hline \multirow[t]{2}{*}{ XR-11006 } & 47,5 & 28 & 35 & 10,6 & 1,25 & 1,25 & 1,21 \\
\hline & & 35 & 35 & 9,3 & 1,00 & & 1,17 \\
\hline \multirow[t]{2}{*}{ TF-VS4 } & 30,0 & 29 & 50 & 12,0 & 1,72 & 1,875 & 1,65 \\
\hline & & 36 & 50 & 6,5 & 1,39 & & 1,56 \\
\hline \multirow[t]{2}{*}{ TQ-15006 } & 47,5 & 19 & 35 & 16,5 & 1,84 & $\left({ }^{2}\right)$ & 0,79 \\
\hline & & 29 & 50 & 17,5 & 1,72 & & 0,86 \\
\hline \multirow[t]{2}{*}{ FL-6,5 } & 42,0 & 22 & 35 & 14,6 & 1,59 & 1,875 & 1,46 \\
\hline & & 25 & 35 & 9,1 & 1,40 & & 1,44 \\
\hline \multirow[t]{2}{*}{ FL -8} & 40,0 & 12 & 20 & 14,7 & 1,67 & 1,875 & 0,95 \\
\hline & & 13 & 20 & 9,1 & 1,53 & & 1,00 \\
\hline \multirow[t]{2}{*}{ TK-VB4 } & 32,5 & 37 & 50 & 22,6 & 1,35 & $\left({ }^{3}\right)$ & 0,60 \\
\hline & & 47 & 50 & 19,4 & 1,06 & & 0,53 \\
\hline \multirow[t]{2}{*}{ TK-VB5 } & 30,0 & 35 & 50 & 18,9 & 1,43 & $\left({ }^{3}\right)$ & 0,74 \\
\hline & & 45 & 50 & 15,2 & 1,11 & & 0,78 \\
\hline \multirow[t]{2}{*}{ TK-VB7,5 } & 30,0 & 36 & 50 & 39,9 & 1,39 & $\left({ }^{3}\right)$ & - \\
\hline & & 46 & 50 & 15,0 & 1,09 & & 0,56 \\
\hline
\end{tabular}

$\left({ }^{1}\right)$ Recomendações da Spraying Systems Co. (1994); $\left({ }^{2}\right)$ Recomendadas para aplicações dirigidas em pósemergência com pingentes; $\left(^{3}\right)$ Não existe uma altura normal para as pontas "Floodjet". Levantar ou abaixar a barra pulverizadora...ou girar os corpos dos bicos, para dobrar a sobreposição dos perfis e conseguir uma ótima cobertura ao longo da barra pulverizadora. 
GALLI, J.C.; MATUO, T.; SIQUEIRA, E.C. Padrão de distribuição de alguns bicos hidráulicos. Planta Daninha, Piracicaba, v.6, n.2, p.144-150, 1983.

MATUO, T. Tecnologia de aplicação de defensivos. In: GRAZIANO NETO, F. ed. Uso de agrotóxico e receituário agronômico. São Paulo, Agroedições, 1982. P.103-106.

MATUO, T.; PERESSIN, V.A.; PERECIN, D.; PIO, L.C.; BRAZ, B.A. Aplicação em jato dirigido em cana-de-açúcar. I. Espaçamento entre bicos TwinJet para aplicação do herbicida MSMA. Eng. Agríc., Campinas, v.14, p.69-82, 1994.

NATION, H.J. The dinamic behaviour of field sprayer booms. J. Agric. Eng. Res., London, v.36, p.61-70, 1982.

ORGANZACIÓN MUNDIAL DE LA SALUD. Material de lucha contra los vectores. Ginebra, 1976. 189p.

PERECIN, D.; PERESSIN, V.A.; MATUO, T.; BARBOSA, J.C.; PIO, L.C.; BRAZ, B.A. Padrões de distribuição obtidos com bicos TwinJet, em função da altura e do espaçamento entre bicos. Eng. Agríc, Campinas, v.14, p.19-30, 1994.

PERECIN, D.; PERESSIN, V.A.; MATUO, T.; BRAZ,B.A.\& PIO, L.C. Padrões de distribuição obtidos com bicos TF-VS4, TJ60-11006 e TQ15006 em mesa de prova. Pesqui. Agropecu. Bras., Brasília, v.33,n.2, p.175-82, 1998.

PERESSIN, V.A.; MATUO, T.; PERECIN, D.; PIO, L.C.; BRAZ, B.A. Aplicação em jato dirigido em cana-de-açúcar. II. Espaçamento entre bicos turbo floodjet para a aplicação de herbicidas. Planta Daninha, Londrina, v.14, p. 86-92, 1996.

ROTH, L.O.; WHITNEY, R.W.; KUHLMAN, D.K. Application uniformity and some nonsymetrical distribuition patterns of agricultural chemicals. Trans. Am. Soc. Agric. Eng, St. Joseph, v.28, n.1, p.47-50, 1985.

SARTORI, S. Pulverizadores para aplicação terrestre tratorizada. In: SIMPÓSIO BRASILEIRO SOBRE TECNOLOGIA DE APLICAÇÃO DE DEFENSIVOS AGRÍCOLAS, 1., 1985. Jaboticabal Anais... Jaboticabal: FUNEP, 1985. p. 47-79.

SILVA, J.G. da. Equipamentos e métodos de aplicação de herbicidas. Informe Agropecuário, Belo Horizonte, v.8, n.87, p.44-54, 1982.

SPRAYING SYSTEMS CO. Teejet: Produtos de pulverização para agricultura. Wheaton, Spraying Systems Co, Manual CAT. 44M-P, 1994. 80p.

VELINI E.D.; et al. Procedimentos para instalação, avaliação e análise de experimentos com herbicidas. Londrina, Sociedade Brasileira da Ciência das Plantas Daninhas, 1995. 42p.

WOLF, D.D. \& SMITH, E.S. Uniformity of seed and fertilizer distribution with a handoperated spinning spreader. Trans. Am. Soc. Agric. Eng., St. Joseph, v.22, n.4, p.761-762, 1979. 